\title{
UV Harmonic Generation and Laser-Driven Proton Acceleration from Thin-Foil Target
}

\author{
Akito SAGISAKA, ${ }^{1}$ Alexander S. PIROZHKOV, ${ }^{1}$ Mamiko NISHIUCHI, ${ }^{1}$ Koichi OGURA, ${ }^{1}$ \\ Hironao SAKAKI, ${ }^{1}$ Anatoly Ya. FAENOV, ${ }^{2,3}$ Tatiana A. PIKUZ, ${ }^{3,4}$ Timur Zh. ESIRKEPOV, ${ }^{1}$ \\ Sergei V. BULANOV ${ }^{1,5}$ Masaki KANDO, ${ }^{1}$ Hiromitsu KIRIYAMA, ${ }^{1}$ and Kiminori KONDO \\ ${ }^{1}$ Kansai Photon Science Institute, National Institutes for Quantum and Radiological Science and Technology, \\ 8-1-7 Umemidai, Kizugawa-city, Kyoto 619-0215 \\ ${ }^{2}$ Open and Transdisciplinary Research Initiatives, Osaka University, Suita, Osaka 565-0871 \\ ${ }^{3}$ Joint Institute for High Temperatures, Russian Academy of Sciences, Moscow 125412, Russia \\ ${ }^{4}$ PPC and Graduate School of Engineering, Osaka University, 2-1 Yamadaoka, Suita, Osaka 565-0871 \\ ${ }^{5}$ Institute of Physics ASCR, v.v.i.(FZU), ELI-Beamlines Project, 18221 Prague, Czech Republic
}

(Received December 11, 2017)

\begin{abstract}
UV harmonics and protons are observed with a high-intensity Ti:sapphire laser. High-energy protons are generated with an aluminum thin-foil target. Simultaneously with the high-energy protons the generation of third- and fourth-order harmonics are observed in the reflection direction by using a high-intensity high-contrast laser.
\end{abstract}

Key Words: High-intensity laser, Harmonic generation, Proton acceleration

\section{Introduction}

In high-intensity laser interactions with plasmas high-energy particles, $\mathrm{x}$-rays, and high-order harmonics are produced. ${ }^{1-6)}$ High-energy protons have been observed at the rear side of thin-foil targets. ${ }^{2-4}$ High-order harmonics generated from the solid surface in the direction of the specular reflection are observed by optimizing the preformed plasma at the front side of the target. Zepf et al. have demonstrated the importance of clean rising edge in the laser pulse for high-order harmonic generation. ${ }^{7)}$ A series of harmonics are observed with plasma mirrors that suppress the prepulse of the high-intensity laser. ${ }^{8-14)}$ The high order harmonics have been observed in the relativistic regime. ${ }^{12-15)}$ Models which can explain harmonic generation in the strongly relativistic regime include the relativistic oscillating mirror model ${ }^{6,16,17)}$ and the sliding mirror model. ${ }^{18,19)}$ In these models, the oscillating overdense layer of plasma electrons reflects the incoming laser pulse. The reflected laser pulse is modulated and contains high-order harmonics. The laser and thin-foil interaction can generate high-energy protons on the rear side and UV harmonics on the front side simultaneously. The preformed plasma affects absorption of the main laser pulse $\mathrm{e}^{20)}$ and, in particular, high-energy proton generation. ${ }^{21,22)}$ The UV harmonics also decrease due to the generation of preformed plasma. In order to observe the simultaneous generation of the higher-energy protons and high-order harmonics, it is necessary to increase the contrast ratio between the main laser pulse and the ASE level. Harmonics are useful tools to diagnose such things as electron density or magnetic fields in high-density plasmas. $^{23-27)}$ Tatarakis et al. and Wagner et al. have reported using the reflected harmonics for the measurements of magnetic fields. ${ }^{25,26)}$ In this experiment, we observe UV harmonics up to the fourth order simultaneously with protons using an aluminum foil target irradiated with a high-contrast Ti:sapphire laser.

\section{Experimental setup}

We use the Ti:sapphire laser system named J-KAREN at National Institutes for Quantum and Radiological Science and Technology (QST) ${ }^{28,29)}$ as a high-intensity, high-contrast laser. The pulse duration and central wavelength of the laser are $\sim 35$ fs [full width at half maximum (FWHM)] and $\sim 0.8 \mu \mathrm{m}$ for this experiment, respectively. The contrast ratio of the ASE is $\sim 10^{-10}$. Figure 1 shows the schematic view of the experimental setup. A p-polarized laser beam is focused by an off-axis parabolic mirror with a focal length of $\sim 320 \mathrm{~mm}$ to the target at an incidence angle of $45^{\circ}$. The spot size of the focused laser beam is $\sim 3 \mu \mathrm{m}$ (FWHM). The estimated peak intensity is up to $\sim 10^{21} \mathrm{~W} / \mathrm{cm}^{2}$; for the correct peak intensity estimate, ${ }^{30)}$ we use the effective pulse duration ( $\left.40 \mathrm{fs}\right)$ and effective spot radius $(\sim 2.5 \mu \mathrm{m})$. The protons are observed with a stack of track detectors consisting of alternating layers of radiochromic film (RCF) and $\mathrm{CR}-39$ in the direction normal to

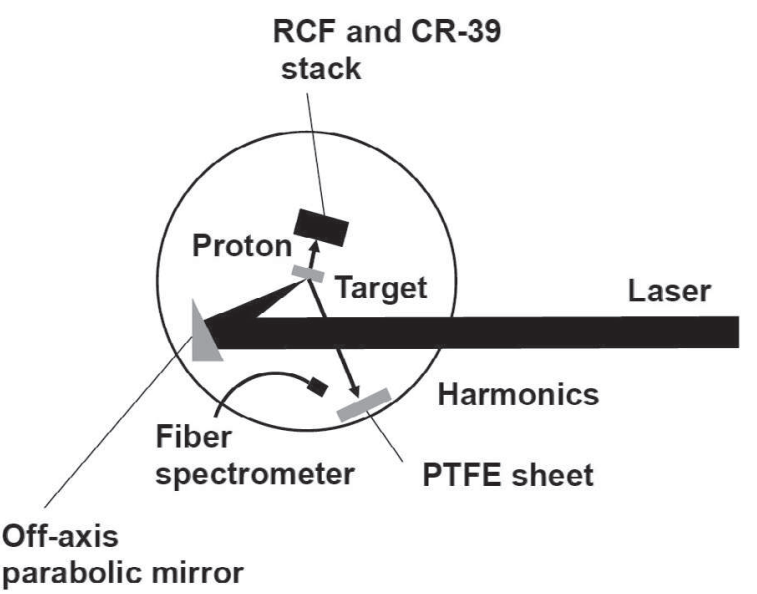

Fig. 1 Schematic diagram of the experimental setup. 
the target. ${ }^{31,32)}$ The stack is placed at a distance of $\sim 55 \mathrm{~mm}$ from the target. The reflected UV harmonics are simultaneously observed with a fiber spectrometer (Ocean Optics: USB2000). ${ }^{33)}$ The specularly reflected harmonic radiation is scattered from a polytetrafluoroethylene (PTFE) sheet ${ }^{20,34)}$ and pass through a fiber and vacuum feed-through. The relative intensity of the measured spectrum is calibrated by a Deuterium lamp. The calibration includes the reflection of the PTFE sheet, transmission of the fiber and feed-through, and spectrometer.

\section{Experimental results}

Figure 2 (a) shows the proton beam pattern from a $2 \mu \mathrm{m}$ thick aluminum target, recorded with a RCF detector (the numbers in the images refer to stack position and the size is $70 \mathrm{~mm} \times 70 \mathrm{~mm}$ ). The CR-39 detectors are inserted between RCF detectors. The proton energy is up to $\sim 36 \mathrm{MeV}$ by stack number 10. The proton energy of stack number 11 is $\sim 38 \mathrm{MeV}$ for Fig. 2(a). The maximum proton energy of $\sim 38 \mathrm{MeV}$ is observed. Figure 2 (b) shows the reflected UV harmonic spectra. The sharp (1-2 pixels wide) peaks are from hard $\mathrm{x}$-rays produced during the laser-plasma interaction. The third- and fourth-order harmonic peaks are clearly observed; both peaks are broadened and shifted towards the long wavelength side. The red shift of the harmonic spectra can be caused by the Doppler shift at the critical density surface. ${ }^{35-38)}$ The strong ponderomotive force of the main laser pulse pushes the critical density of the expanding preformed plasma. ${ }^{39)}$ The critical density at the wavelength of $800 \mathrm{~nm}$ is $\sim 1.7 \times 10^{21} \mathrm{~cm}^{-3}$, while the relativistically-corrected value for our peak intensity is $\sim 3 \times 10^{22} \mathrm{~cm}^{-3}$. We note that under conditions similar to ours the laser pulse has reached the relativistic critical density region. ${ }^{40}$ For example, the velocity of the reflecting surface is estimated to be $\sim 8 \times 10^{8} \mathrm{~cm} / \mathrm{s}$ from the spectrum shift of $\sim 10 \mathrm{~nm}$ for the third-order harmonic at a reflected angle of $45^{\circ} .{ }^{6,41}$ The broadening of the UV spectra can be attributed to the relativistic self-phase modulation of the incident laser as it propagates through the preformed plasma. ${ }^{42-44)}$ The spectral shift and broadening show that preformed plasma is generated under our conditions at the front side of target. In the case of preformed plasma presence, the position of the harmonic generation requires consideration. We assume that the high-order harmonics are generated in the vicinity of the relativistically-corrected critical density region where the electron

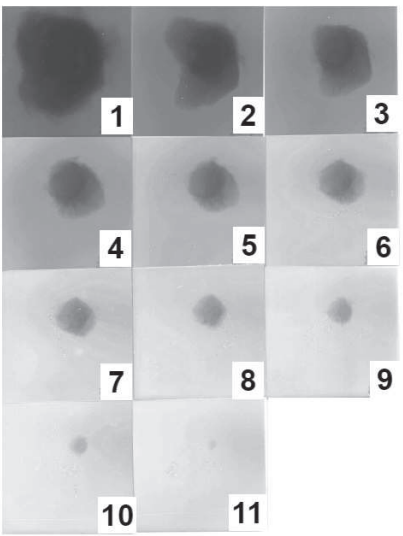

(b)

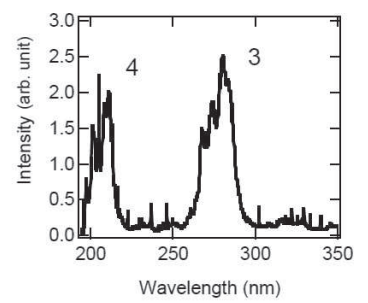

Fig. 2 Proton beam footprints recorded with the RCF stack detector (a) and UV harmonic spectra (b) from the $2 \mu \mathrm{m}$ thick aluminum target. density distribution is highly inhomogeneous. Possible interpretation of harmonic generation is given by the relativistic oscillating mirror. ${ }^{6,16,17)}$ Under our experimental conditions, the ASE effects can result in the formation of a relatively low-density plasma in front of the target. In this case the "relativistic oscillating mirror" corresponds to the oscillations of the relativistic critical surface. UV harmonics and high-energy protons are observed simultaneously with a high-contrast laser.

Figure 3 shows the harmonic spectra for $1.5 \mu \mathrm{m}$ thick (a) and $0.8 \mu \mathrm{m}$ thick (b) aluminum targets. The UV harmonics up to the fourth order are observed for both target thicknesses. Under these conditions, maximum proton energies of $\sim 38 \mathrm{MeV}$ (a) and $\sim 39 \mathrm{MeV}$ (b) are observed. Both UV harmonics and high-energy protons are also observed simultaneously for the thinner target $(0.8 \mu \mathrm{m})$.

Figure 4 shows the integrated third-order harmonic signal as a function of the deflection angle of the proton beam for a $0.8 \mu \mathrm{m}$ thick aluminum target obtained from separate laser shots having various fluctuations. The deflection angle is defined as the difference between the target normal direction and proton beam direction with proton energies of $\sim 34 \mathrm{MeV}$ to $\sim 36 \mathrm{MeV}$. Vertical and horizontal bars correspond to error bars and width of the proton beam pattern, respectively. The difference between the right and left sides of the bars arises due to the proton beam profile asymmetry. The right side of the proton beam width of the lowest THG signal in Fig. 4 is cut by the edge of the RCF detector. When the deflection angle is changed, the peaks of the third- and fourth-order harmonics also change. The third-order harmonic signal decreases with the deflection angle of the proton beam which tends towards the laser beam direction. The proton beam can be deflected due to several effects, such as prepulses producing a near critical plasma channel, ${ }^{45)}$ deformation of the rear side of the target, ${ }^{4-48)}$ relativistic effects at the oblique incidence, ${ }^{49)}$ and so on. The facts that the UV harmonics are observed in the specular direction, and that their yield is related to the de- (a)

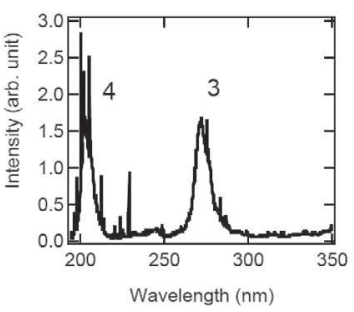

(b)

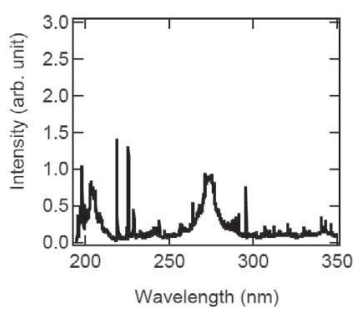

Fig. 3 Spectra of reflected UV harmonics with the aluminum target thickness of (a) $1.5 \mu \mathrm{m}$ and (b) $0.8 \mu \mathrm{m}$.

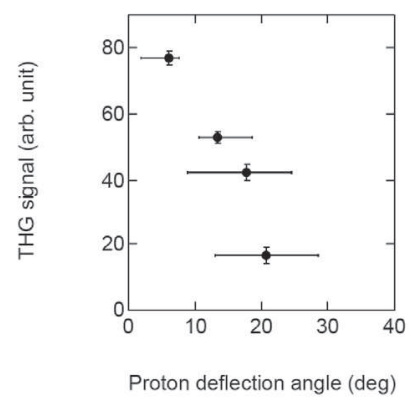

Fig. 4 Integrated third-order harmonic signal as a function of the deflection angle of the proton beam for the $0.8 \mu \mathrm{m}$ thick aluminum target. 
flection of the proton beam rather than the proton energy, suggest that the relevant reason is probably the rear surface deformation from the laser prepulse. In this assumption the UV harmonic intensity probably decreases as the front surface preformed plasma and target bending increases, where the change of the preformed plasma related to the fluctuation of the prepulse condition is considered. The combination of measurements (UV harmonics and protons) seems to be useful for the diagnostics of the laser and plasma interaction.

\section{Summary}

We obtained experimentally the simultaneous generation of UV harmonics and high-energy protons from aluminum foil targets. The peaks of the third- and fourth-order harmonics are broadened for a $2 \mu \mathrm{m}$ thick aluminum target. The spectra broadening indicates preformed plasma generation at the front side of the target. The UV harmonic intensity decreases as the proton deflection angle increases with the $0.8 \mu \mathrm{m}$ thick aluminum target. The proton beam as deflected from the target normal direction towards the laser beam direction; this deflection is most probably caused by the deformation of the target rear surface. Combining measurements of UV harmonics and protons appears to be useful for the diagnostics of the laser and plasma interaction. The high contrast pulse can generate both UV harmonics and high-energy protons.

\section{Acknowledgments}

We acknowledge the support of this work by the Kansai Photon Science Institute, National Institutes for Quantum and Radiological Science and Technology (QST). SVB acknowledges support from the project High Field Initiative (CZ.02.1.01/0.0/0.0/15 003/0000449) from European Regional Development Fund.

\section{References}

1) G. A. Mourou, T. Tajima, and S. V. Bulanov: Rev. Mod. Phys. 78 (2006) 309

2) M. Borghesi, J. Fuchs, S. V. Bulanov, A. J. Mackinnon, P. K. Patel, and M. Roth: Fusion Sci. Technol. 49 (2006) 412.

3) H. Daido, M. Nishiuchi, and A. S. Pirozhkov: Rep. Prog. Phys. 75 (2012) 056401.

4) A. Macchi, M. Borghesi, and M. Passoni: Rev. Mod. Phys. 85 (2013) 751 .

5) U. Teubner and P. Gibbon: Rev. Mod. Phys. 81 (2009) 445.

6) S. V. Bulanov, T. Zh. Esirkepov, M. Kando, A. S. Pirozhkov, and N. N. Rosanov: Phys. Usp. 56 (2013) 429.

7) M. Zepf, G. D. Tsakiris, G. Pretzler, I. Watts, D. M. Chambers, P. A. Norreys, U. Andiel, A. E. Dangor, K. Eidmann, C. Gahn, et al.: Phys. Rev. E 58 (1998) R5253.

8) P. Monot, G. Doumy, S. Dobosz, M. Perdrix, P. D’Oliveira, F. Quéré, F. Réau, P. Martin, P. Audebert, J.-C. Gauthier, et al.: Opt. Lett. 29 (2004) 893.

9) G. Doumy, S. Dobosz, P. D'Oliveira, P. Monot, M. Perdrix, F. Quéré, F. Réau, Ph. Martin, P. Audebert, J.-C. Gauthier, et al.: Appl. Phys. B 78 (2004) 901.

10) F. Quéré, C. Thaury, P. Monot, S. Dobosz, Ph. Martin, J.-P. Geindre, and P. Audebert: Phys. Rev. Lett. 96 (2006) 125004.

11) A. Lévy, T. Ceccotti, P. D’Oliveira, F. Réau, M. Perdrix, F. Quéré, P. Monot, M. Bougeard, H. Lagadec, P. Martin, et al.: Opt. Lett. 32 (2007) 310 .

12) B. Dromey, M. Zepf, A. Gopal, K. Lancaster, M. S. Wei, K. Krushelnick, M. Tatarakis, N. Vakakis, S. Moustaizis, R. Kodama, et al.: Nature Phys. 2 (2006) 456.

13) B. Dromey, S. Kar, C. Bellei, D. C. Carroll, R. J. Clarke, J. S. Green, S. Kneip, K. Markey, S. R. Nagel, P. T. Simpson, et al.: Phys. Rev. Lett. 99 (2007) 085001.
14) C. Thaury, F. Quéré, J.-P. Geindre, A. Levy, T. Ceccotti, P. Monot, M. Bougeard, F. Réau, P. D'Oliveira, P. Audebert, et al.: Nature Phys. 3 (2007) 424.

15) A. Tarasevitch, K. Lobov, C. Wünsche, and D. V. D. Linde: Phys Rev. Lett. 98 (2007) 103902.

16) S. V. Bulanov, N. M. Naumova, and F. Pegoraro: Phys. Plasmas 1 (1994) 745.

17) R. Lichters, J. Meyer-ter-Vehn, and A. Pukhov: Phys. Plasmas 3 (1996) 3425

18) A. S. Pirozhkov, S. V. Bulanov, T. Z. Esirkepov, M. Mori, A. Sagisaka, and H. Daido: Phys. Plasmas 13 (2006) 013107.

19) A. S. Pirozhkov, S. V. Bulanov, T. Z. Esirkepov, M. Mori, A. Sagisaka, and H. Daido: Phys. Lett. A 349 (2006) 256.

20) A. S. Pirozhkov, I. W. Choi, J. H. Sung, S. K. Lee, T. J. Yu, T. M. Jeong, I. J. Kim, N. Hafz, C. M. Kim, K. H. Pae, et al.: Appl. Phys. Lett. 94 (2009) 241102.

21) A. Sagisaka, H. Nagatomo, H. Daido, A. S. Pirozhkov, K. Ogura, S. Orimo, M. Mori, M. Nishiuchi, A. Yogo, and M. Kado: J. Plasma. Phys. 75 (2009) 609.

22) T. Zh. Esirkepov, J. K. Koga, A. Sunahara, T. Morita, M. Nishikino, K. Kageyama, H. Nagatomo, K. Nishihara, A. Sagisaka, H. Kotaki, et al.: Nucl. Instrum. Methods Phys. Res. A 745 (2014) 150.

23) D. Descamps, C. Lyngå, J. Norin, A. L’Huillier, C.-G. Wahlström, J.-F. Hergott, H. Merdji, P. Salières, M. Bellini, and T. W. Hänsch: Opt. Lett. 25 (2000) 135.

24) W. Theobald, R. Hä $\beta$ ner, C. Wülker, and R. Sauerbrey: Phys. Rev. Lett. 77 (1996) 298.

25) M. Tatarakis, A. Gopal, I. Watts, F. N. Beg, M.-S. Wei, A. E. Dangor, K. Krushelnick, U. Wagner, P. A. Norreys, E. L. Clark, et al.: Phys. Plasmas 9 (2002) 2244.

26) U. Wagner, M. Tatarakis, A. Gopal, F. N. Beg, E. L. Clark, A. E. Dangor, R. G. Evans, M. G. Haines, S. P. D. Mangles, P. A. Norreys, et al.: Phys. Rev. E 70 (2004) 026401.

27) K. Krushelnick, I. Watts, M. Tatarakis, A. Gopal, U. Wagner, F. N. Beg, E. L. Clark, R. J. Clarke, A. E. Dangor, P. A. Norreys, et al.: Plasma Phys. Control. Fusion 44 (2002) B233.

28) H. Kiriyama, M. Mori, Y. Nakai, T. Shimomura, H. Sasao, M. Tanoue, S. Kanazawa, D. Wakai, F. Sasao, H. Okada, et al.: Opt. Lett. 35 (2010) 1497.

29) H. Kiriyama, M. Mori, A. S. Pirozhkov, K. Ogura, A. Sagisaka, A. Kon, T. Zh. Esirkepov, Y. Hayashi, H. Kotaki, M. Kanasaki, et al.: IEEE Sel. Topics J. Quantum Electron. 21 (2015) 1601118.

30) A. S. Pirozhkov, Y. Fukuda, M. Nishiuchi, H. Kiriyama, A. Sagisaka, K. Ogura, M. Mori, M. Kishimoto, H. Sakaki, N. P. Dover, et al.: Opt. Express 25 (2017) 20486.

31) K. Ogura, M. Nishiuchi, A. S. Pirozhkov, T. Tanimoto, A. Sagisaka, T. Zh Esirkepov, M. Kando, T. Shizuma, T. Hayakawa, H. Kiriyama, et al.: Opt. Lett. 37 (2012) 2868.

32) M. Nishiuchi, H. Sakaki, T. Zh. Esirkepov, K. Nishio, T.A. Pikuz, A. Ya. Faenov, I.Yu. Skobelev, R. Orlandi, H. Sako, A. S. Pirozhkov, et al.: Phys. Plasmas 22 (2015) 033107.

33) I. W. Choi, I. J. Kim, K. H. Pae, K. H. Nam, C.-L. Lee, H. Yun, H. T. Kim, S. K. Lee, T. J. Yu, J. H. Sung, et al.: Appl. Phys. Lett. 99 (2011) 181501

34) A. S. Pirozhkov, I. W. Choi, J. H. Sung, S. K. Lee, T. J. Yu, T. M. Jeong, I. J. Kim, N. Hafz, C. M. Kim, K. H. Pae, et al.: AIP Conf. Proc. 1153 (2009) 7.

35) M. Zepf, M. Castro-Colin, D. Chambers, S. G. Preston, J. S. Wark, J. Zhang, C. N. Danson, D. Neely, P. A. Norreys, A. E. Dangor, et al.: Phys. Plasmas 3 (1996) 3242.

36) M. P. Kalashnikov, P. V. Nickles, Th. Schlegel, M. Schnuerer, F. Billhardt, I. Will, W. Sandner, and N. N. Demchenko: Phys. Rev. Lett. 73 (1994) 260.

37) S. C. Wilks, W. L. Kruer, M. Tabak, and A. B. Langdon: Phys. Rev. Lett. 69 (1992) 1383.

38) W. L. Kruer, E. J, Valeo, and K. G. Estabrook: Phys. Rev, Lett. 35 (1975) 1076.

39) A. Sagisaka, H. Daido, K. Ogura, S. Orimo, Y. Hayashi, M. Nishiuchi, M. Mori, K. Matsukado, A. Fukumi, Z. Li, et al.: Appl. Phys. B 78 (2004) 919.

40) M. A. Alkhimova, A. Ya Faenov, I. Yu Skobelev, T. A. Pikuz, M. Nishiuchi, H. Sakaki, A. S. Pirozhkov, A. Sagisaka, N. P. Dover, K. Kondo, et al.: Opt. Express 25 (2017) 29501. 
41) A. S. Pirozhkov, J. Ma, M. Kando, T. Zh. Esirkepov, Y. Fukuda, L.-M. Chen, I. Daito, K. Ogura, T. Homma, Y. Hayashi, H. Kotaki, et al.: Phys. Plasmas 14 (2007) 123106.

42) C. A. Coverdale, C. B. Darrow, C. D. Decker, N. M. Naumova, T. Zh. Esirkepov, A. S. Sakharov, S. V. Bulanov, W. B. Mori, and K. C. Tzeng: Fiz. Plazmy 22 (1996) 685; [ibid Plasma Phys. Rep. 22 (1996) 617]

43) Y. R. Shen: The Principles of Nonlinear Optics (New York: Wiley, 1984)

44) I. Watts, M. Zepf, E. L. Clark, M. Tatarakis, K. Krushelnick, A. E. Dangor, R. Allott, R. J. Clarke, D. Neely, and P. A. Norreys: Phys. Rev. E 66, 036409 (2002)
45) A. Yogo, H. Daido, S. V. Bulanov, K. Nemoto, Y. Oishi, T. Nayuki, T. Fujii, K. Ogura, S. Orimo, A. Sagisaka, et al.: Phys. Rev. E 77 (2008) 016401.

46) F. Lindau, O. Lundh, A. Persson, P. McKenna, K. Osvay, D. Batani, and C.-G. Wahlström: Phys. Rev. Lett. 95 (2005)175002.

47) O. Lundh, F. Lindau, A. Persson, C.-G. Wahlström, P. McKenna, and D. Batani: Phys. Rev. E 76 (2007) 026404.

48) D. Batani, R. Jafer, M. Veltcheva, R. Dezulian, O. Lundh, F. Lindau, A. Persson, K. Osvay, C.-G. Wahlström, D. C. Carroll, et al.: New J. Phys. 12 (2010) 045018.

49) T. Morita, T. Zh. Esirkepov, S.V. Bulanov, J. Koga, and M. Yamagiwa: Phys. Rev. Lett. 100 (2008) 145001. 